\title{
Tensor renormalization group approach to higher dimensional fermions
}

\section{Ryo Sakai*}

Institute for Theoretical Physics, Kanazawa University, Kanazawa 920-1192, Japan

E-mail: sakaidhep.s.kanazawa-u.ac.ip

\section{Shinji Takeda ${ }^{\dagger}$}

Institute for Theoretical Physics, Kanazawa University, Kanazawa 920-1192, Japan

E-mail: Eakedadhep.s.kanazawa-u.ac.jp

We apply the higher order tensor renormalization group to two and three dimensional lattice fermion systems. To deal with tensor networks including Grassmann variables, we introduce Grassmann higher order tensor renormalization group (GHOTRG). Thanks to its deterministic property, GHOTRG is perfectly free of the sign problem. To test the validity of the new algorithm, we analyze well-known systems such as the Thirring model using GHOTRG.

34th annual International Symposium on Lattice Field Theory

24-30 July 2016

University of Southampton, UK

\footnotetext{
* Speaker.

${ }^{\dagger}$ This work was supported by Kanazawa University SAKIGAKE Project and by MEXT as "Exploratory Challenge on Post-K computer" (Frontiers of Basic Science: Challenging the Limits).
} 


\section{Introduction}

Monte Carlo simulation cannot be simply applied to systems whose Boltzmann weight is complex, for example, finite density fermion systems or $\theta$ term included systems, known as the complex action problem (the sign problem). One of possibilities to avoid the sign problem is to use the tensor renormalization group (TRG) [W] whose algorithm is applicable regardless of whether the Boltzmann weight is complex or not.

What the TRG can compute is, for instance, a partition function of classical statistical systems or that in the path integral representation of quantum field theory. The first step of TRG is to represent a partition function of a system in terms of tensor networks. After this step, the partition function can be expressed by contraction of a bunch of tensors. The second step is to perform a coarse-graining of the tensor network by using the singular value decomposition to preserve important information of the system while keeping an efficiency of the calculation. By repeating the coarse-graining, one can gradually reduce the number of tensors and then it is feasible to perform the full contraction of a few renormalized tensors, and finally one can obtain the partition function approximately. Although the original idea of TRG introduced by Levin and Nave [四] was limited to two dimensional systems, a new coarse-graining method suited for any higher dimensional system was proposed in Ref. [■]. The new method is based on the higher order singular value decomposition, thus it is called higher order tensor renormalization group (HOTRG).

Our ultimate goal is to apply the idea of TRG approach to lattice QCD which is a relativistic four dimensional non-Abelian gauge theory coupled with the quark fields. The tensor network representation of non-Abelian gauge theories was already attempted in Ref. [B] and the HOTRG for boson systems was already done. Thus, the final missing piece to achieve the goal is a treatment of fermions in higher dimensions. In this report, we will formulate HOTRG for fermions in higher dimensional systems, we call this Grassmann higher order tensor renormalization group (GHOTRG). As a concrete example, we will provide a detail of GHOTRG in two dimensions.

\section{Grassmann higher order tensor renormalization group}

In this section, after introducing the model and the notation, we briefly review the HOTRG for bosonic tensors. Then we explain the essence of GHOTRG in two dimensional systems. Lattice units $a=1$ are assumed in the following.

\subsection{Model and notation in two dimensional systems}

Following the prescription described in Refs. [四, [] , one can obtain the tensor network representation of the partition function of relativistic fermions on a lattice

$$
\begin{aligned}
Z & =\int \mathcal{D} \psi \mathcal{D} \bar{\psi} e^{-\sum_{n} \mathcal{L}_{n}} \\
& =\operatorname{Tr}_{\{x, t\}} \int \prod_{n} \mathcal{T}_{x_{n} t_{n} x_{n-1} t_{n-\hat{2}}},
\end{aligned}
$$

where $n$ is the lattice coordinate, the fermion fields $\psi, \bar{\psi}$ are two-component spinors, and $m$ and $\mu$ denote the mass and the chemical potential respectively. The tensor $\mathcal{T}$, an elementary building 
block of the tensor network, is defined as

$$
\begin{aligned}
\mathcal{T}_{x_{n} t_{n} x_{n-1} t_{n-\hat{2}}}= & T_{x_{n} t_{n} x_{n-1} t_{n-2}} \mathrm{~d} \bar{\eta}_{n, 2}^{x_{n, 2}} \mathrm{~d} \eta_{n, 1}^{x_{n, 1}} \mathrm{~d} \bar{\xi}_{n, 2}^{t_{n, 2}} \mathrm{~d} \xi_{n, 1}^{t_{n, 1}} \mathrm{~d} \eta_{n, 2}^{x_{n-\hat{1}, 2}} \mathrm{~d} \bar{\eta}_{n, 1}^{x_{n-\hat{1}, 1}} \mathrm{~d} \xi_{n, 2}^{t_{n-\hat{2}, 2}} \mathrm{~d} \bar{\xi}_{n, 1}^{t_{n-\hat{2}, 1}} \\
& \cdot\left(\bar{\eta}_{n+\hat{1}, 1} \eta_{n, 1}\right)^{x_{n, 1}}\left(\bar{\eta}_{n, 2} \eta_{n+\hat{1}, 2}\right)^{x_{n, 2}}\left(\bar{\xi}_{n+\hat{2}, 1} \xi_{n, 1}\right)^{t_{n, 1}}\left(\bar{\xi}_{n, 2} \xi_{n+\hat{2}, 2}\right)^{t_{n, 2}},
\end{aligned}
$$

where the original fermion fields $\psi, \bar{\psi}$ have been already integrated out, instead the another set of Grassmann variables $\eta_{n, i}, \bar{\eta}_{n, i} \xi_{n, i}$ and $\bar{\xi}_{n, i}(i=1,2)$ have been introduced and they satisfy

$$
\int \mathrm{d} \eta_{n, i} \eta_{n, i}=\int \mathrm{d} \bar{\eta}_{n, i} \bar{\eta}_{n, i}=\int \mathrm{d} \xi_{n, i} \xi_{n, i}=\int \mathrm{d} \bar{\xi}_{n, i} \bar{\xi}_{n, i}=1, \quad \text { for } i=1,2 .
$$

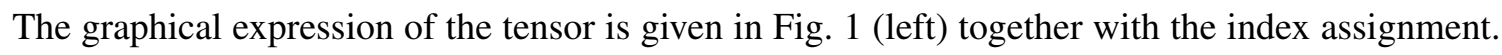
In Eq. (L2.2), $T_{x_{n} t_{n} x_{n-1} t_{n-\hat{2}}}$ is a normal tensor whose components are just numbers and explicitly given in Refs. [四, []] for two types of interaction, the Schwinger model and the $N_{\mathrm{f}}=1$ Gross-Neveu model (the Thirring model) in two dimensions. We call $T_{x_{n} t_{n} x_{n-1} t_{n-\hat{2}}}$ bosonic tensor and the rest of $\mathcal{T}$ composed of the Grassmann variables

$$
\begin{aligned}
& \mathrm{d} \bar{\eta}_{n, 2}^{x_{n, 2}} \mathrm{~d} \eta_{n, 1}^{x_{n, 1}} \mathrm{~d} \bar{\xi}_{n, 2}^{t_{n, 2}} \mathrm{~d} \xi_{n, 1}^{t_{n, 1}} \mathrm{~d} \eta_{n, 2}^{x_{n-1}, 2} \mathrm{~d} \bar{\eta}_{n, 1}^{x_{n-1} 1} \mathrm{~d} \xi_{n, 2}^{t_{n-2}} \mathrm{~d} \bar{\xi}_{n-1}^{t_{n-1}} \\
& \cdot\left(\bar{\eta}_{n+\hat{1}, 1} \eta_{n, 1}\right)^{x_{n, 1}}\left(\bar{\eta}_{n, 2} \eta_{n+\hat{1}, 2}\right)^{x_{n, 2}}\left(\bar{\xi}_{n+\hat{2}, 1} \xi_{n, 1}\right)^{t_{n, 1}}\left(\bar{\xi}_{n, 2} \xi_{n+\hat{2}, 2}\right)^{t_{n, 2}}
\end{aligned}
$$

is called Grassmann part. Note that the indices in LHS of Eq. (2.2) should be read as $x_{n}=\left(x_{n, 1}, x_{n, 2}\right)$ and $t_{n}=\left(t_{n, 1}, t_{n, 2}\right)$, reflecting the fact that the original Grassmann variable $\psi$ has two components. Each index runs from 0 to 1 , thus the tensor

$$
\mathcal{T}_{x_{n} t_{n} x_{n-1} t_{n-\hat{2}}}=\mathcal{T}_{\left(x_{n, 1}, x_{n, 2}\right)\left(t_{n, 1}, t_{n, 2}\right)\left(x_{n-\hat{1}, 1}, x_{n-\hat{1}, 2}\right)\left(t_{n-\hat{2}, 1}, t_{n-\hat{2}, 2}\right)}
$$

has $2^{2 \times 4}$ elements at the initial stage.

In the following we discuss the renormalization procedure for the bosonic tensor and the Grassmann part separately.

\subsection{Normal HOTRG procedure for bosonic tensors}

In this subsection, let us see the renormalization of bosonic tensors, especially focus on the coarse-graining along the $\hat{1}$-direction. This is a brief review of the normal HOTRG [四].

First we consider a new tensor $M$ by contracting two bosonic tensors placed next to each other along the 1 -direction (see Fig. $\mathbb{D}$ )

$$
M_{x_{n+1} t_{n}^{+} x_{n-1} \hat{1}_{n}^{-}}=\sum_{x_{n}} T_{x_{n} t_{n} x_{n-1} t_{n-2}} T_{x_{n+1} t_{n+\hat{1}} x_{n} t_{n+\hat{1}-\hat{2}}}
$$

where the integrated indices are defined as

$$
t_{n}^{+}=t_{n} \otimes t_{n+\hat{1}}, \quad t_{n}^{-}=t_{n-\hat{2}} \otimes t_{n+\hat{1}-\hat{2}} .
$$

And then one defines $M^{+}$and $M^{-}$as

$$
M_{t_{n}^{ \pm}, t_{n}^{ \pm}}^{ \pm}=\sum_{x_{n+\hat{1}}, x_{n-\hat{1}}, t_{n}^{\mp}} M_{\left(t_{n}^{ \pm}\right),\left(x_{n+\hat{1}} x_{n-1} t_{n}^{\mp}\right)}^{\prime} M_{\left(x_{n+\hat{1}} x_{n-1} t_{n}^{\mp}\right),\left(t_{n}^{ \pm \prime}\right)}^{\prime \dagger},
$$


with

$$
M_{\left(t_{n}^{ \pm}\right),\left(x_{n+\hat{1}} x_{n-1} t_{n}^{\mp}\right)}^{\prime}=M_{x_{n+1} t_{n}^{+} x_{n-1} t_{n}^{-}} .
$$

Next we apply the eigenvalue decomposition to $M^{+}$and obtain a unitary matrix $U^{+}$and the eigenvalues $\lambda^{+}$:

$$
M_{t_{n}^{+}, t_{n}^{+\prime}}^{+}=\sum_{t_{n^{*}, \mathrm{~b}}} U_{t_{n}^{+}, t_{n^{*}, \mathrm{~b}}}^{+} \lambda_{t_{n^{*}, \mathrm{~b}}^{+}}^{+} U_{t_{n^{*}, \mathrm{~b}}, t_{n}^{+^{\prime}}}^{+}
$$

where the new index $t_{n^{*}, \mathrm{~b}}$ is regarded as the second component of the index of the new tensor $T^{\text {new }}$, as it will be clear soon. Similarly one can make $U^{-}$and $\lambda^{-}$from $M^{-}$and then we define $\varepsilon^{+}$and $\varepsilon^{-}$as

$$
\varepsilon^{ \pm}=\sum_{i>D_{\mathrm{cut}}} \lambda_{i}^{ \pm}
$$

with a given $D_{\text {cut }}$ which one can choose. They indicate an amount of truncation error and are used to select the unitary matrix which maintains better precision, say if $\varepsilon^{+}<\varepsilon^{-}$then $U^{+}$is adopted in the next step and vice versa. Now one obtains the new bosonic tensor by using the selected unitary matrix (denoted by $U$ ) and restricting the new indices $1 \leq t_{n^{*}, \mathrm{~b}}, t_{n^{*}-\hat{2}, \mathrm{~b}} \leq D_{\text {cut }}$ (see Fig. 四),

$$
T_{x_{n+\hat{1}} t_{n^{*}, \mathrm{~b}} x_{n-\hat{1}} t_{n^{*}-\hat{2}, \mathrm{~b}}}^{\text {new }}=\sum_{i, j}^{\text {all }} U_{i t_{n^{*}, \mathrm{~b}}}^{*} M_{x_{n+\hat{1}} i x_{n-\hat{1}} j} U_{j t_{n^{*}-\hat{\mathrm{L}} \mathrm{b}}} .
$$

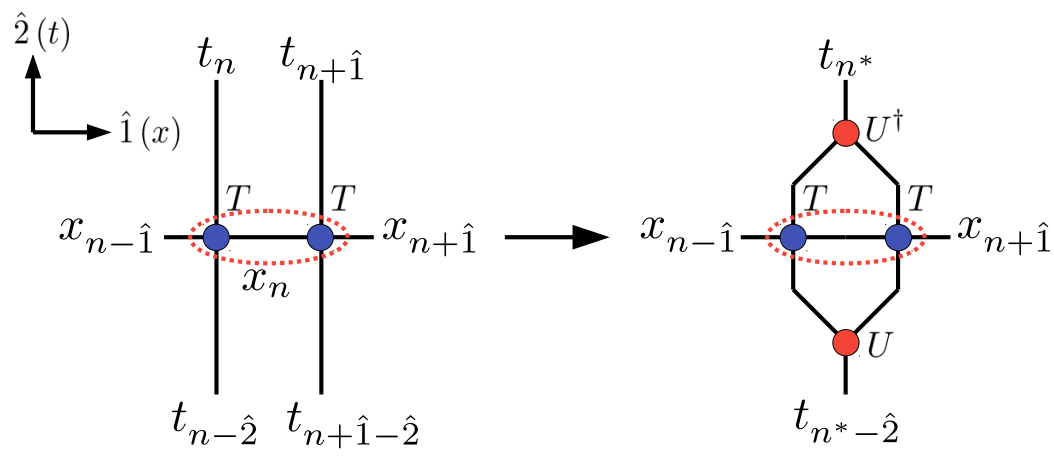

Figure 1: Coarse-graining along the $\hat{1}$-direction.

\subsection{Coarse-graining of the Grassmann part}

Next we explain the coarse-graining of the Grassmann part. Along the $\hat{1}$-direction, the product of the Grassmann part of $\mathcal{T}_{x_{n} t_{n} x_{n-1} t_{n-2}}$

$$
\begin{aligned}
& \mathrm{d} \bar{\eta}_{n, 2}^{x_{n, 2}} \mathrm{~d} \eta_{n, 1}^{x_{n, 1}} \mathrm{~d} \bar{\xi}_{n, 2}^{t_{n, 2}} \mathrm{~d} \xi_{n, 1}^{t_{n, 1}} \mathrm{~d} \eta_{n, 2}^{x_{n-\hat{1}, 2}} \mathrm{~d} \bar{\eta}_{n, 1}^{x_{n-\hat{1}, 1}} \mathrm{~d} \xi_{n, 2}^{t_{n-\hat{2}, 2}} \mathrm{~d} \bar{\xi}_{n, 1}^{t_{n-\hat{2}, 1}} \\
& \cdot\left(\bar{\eta}_{n+\hat{1}, 1} \eta_{n, 1}\right)^{x_{n, 1}}\left(\bar{\eta}_{n, 2} \eta_{n+\hat{1}, 2}\right)^{x_{n, 2}}\left(\bar{\xi}_{n+\hat{2}, 1} \xi_{n, 1}\right)^{t_{n, 1}}\left(\bar{\xi}_{n, 2} \xi_{n+\hat{2}, 2}\right)^{t_{n, 2}}
\end{aligned}
$$


and that of $\mathcal{T}_{x_{n+1} t_{n+\hat{1}} x_{n} t_{n+\hat{1}-\hat{2}}}$ which is located next to $\mathcal{T}_{x_{n} t_{n} x_{n-\hat{1}} t_{n-\hat{2}}}$

$$
\begin{aligned}
& \mathrm{d} \bar{\eta}_{n+\hat{1}, 2}^{x_{n+\hat{1}, 2}} \mathrm{~d} \eta_{n+\hat{1}, 1}^{x_{n+\hat{1}, 1}} \mathrm{~d} \bar{\xi}_{n+\hat{1}, 2}^{t_{n+\hat{1}, 2}} \mathrm{~d} \xi_{n+\hat{1}, 1}^{t_{n+\hat{1}, 1}} \mathrm{~d} \eta_{n+\hat{1}, 2}^{x_{n, 2}} \mathrm{~d} \bar{\eta}_{n+\hat{1}, 1}^{x_{n, 1}} \mathrm{~d} \xi_{n+\hat{1}, 2}^{t_{n+\hat{\imath}, 2}} \mathrm{~d} \bar{\xi}_{n+\hat{1}, 1}^{t_{n+\hat{\imath}, 1}} \\
& \cdot\left(\bar{\eta}_{n+\hat{1}+\hat{1}, 1} \eta_{n+\hat{1}, 1}\right)^{x_{n+\hat{1}, 1}}\left(\bar{\eta}_{n+\hat{1}, 2} \eta_{n+\hat{1}+\hat{1}, 2}\right)^{x_{n+\hat{1}, 2}}\left(\bar{\xi}_{n+\hat{1}+\hat{2}, 1} \xi_{n+\hat{1}, 1}\right)^{t_{n+\hat{1}, 1}}\left(\bar{\xi}_{n+\hat{1}, 2} \xi_{n+\hat{1}+\hat{2}, 2}\right)^{t_{n+\hat{1}, 2}}
\end{aligned}
$$

is reduced to

$$
\begin{aligned}
& (-1)^{x_{n, 1}\left(x_{n, 1}+x_{n, 2}\right)+t_{n, 2}\left(t_{n, 1}+t_{n, 2}\right)+t_{n+\hat{1}, 2}\left(t_{n+\hat{1}, 1}+t_{n+\hat{1}, 2}\right)} \\
& \cdot(-1)^{\left(t_{n, 1}+t_{n, 2}\right)\left(t_{n+\hat{1}, 1}+t_{n+\hat{1}, 2}\right)+\left(t_{n-\hat{2}, 1}+t_{n-\hat{2}, 2}\right)\left(t_{n+\hat{1}-\hat{2}, 1}+t_{n+\hat{1}-\hat{2}, 2}\right)} \\
& \cdot \mathrm{d} \bar{\eta}_{n+\hat{1}, 2}^{x_{n+1}} \mathrm{~d} \eta_{n+\hat{1}, 1}^{x_{n+\hat{1}, 1}} \mathrm{~d} \xi_{n^{*}, \mathrm{f}}^{t^{*}} \mathrm{~d} \eta_{n, 2}^{x_{n-\hat{1}, 2}} \mathrm{~d} \bar{\eta}_{n, 1}^{x_{n-\hat{1}, 1}} \mathrm{~d} \bar{\xi}_{n^{*}-t^{*}, \mathrm{f}}^{t_{n}} \\
& \cdot\left(\bar{\eta}_{n+\hat{1}+\hat{1}, 1} \eta_{n+\hat{1}, 1}\right)^{x_{n+\hat{1}, 1}}\left(\bar{\eta}_{n+\hat{1}, 2} \eta_{n+\hat{1}+\hat{1}, 2}\right)^{x_{n+\hat{1}, 2}}\left(\bar{\xi}_{n^{*}+\hat{2}} \xi_{n^{*}}\right)^{t_{n^{*}, \mathrm{f}}} .
\end{aligned}
$$

In the above step, we have introduced the new index $t_{n^{*}, \mathrm{f}}$ and the Grassmann variables $\xi_{n^{*}}, \bar{\xi}_{n^{*}+\hat{2}}$, $\mathrm{d} \xi_{n^{*}}, \mathrm{~d} \bar{\xi}_{n^{*}+\hat{2}}$ which are defined by

$$
\begin{gathered}
t_{n^{*}, \mathrm{f}}=\left(t_{n, 1}+t_{n, 2}+t_{n+\hat{1}, 1}+t_{n+\hat{1}, 2}\right) \bmod 2, \\
\left(\mathrm{~d} \xi_{n^{*}} \mathrm{~d} \bar{\xi}_{n^{*}+\hat{2}} \bar{\xi}_{n^{*}+\hat{2}} \xi_{n^{*}}\right)^{t_{n^{*}, \mathrm{f}}}(=1) .
\end{gathered}
$$

In this way, by integrating out old d.o.f and replacing them with this new set of Grassmann variables, one can perform a coarse-graining of the Grassmann part. The sign factor in Eq. (2.14) arises because of the anticommutative property of Grassmann variables. This factor is incorporated into the new bosonic tensor $T_{x_{n+1} t_{n^{*}, b} x_{n-1} t_{n^{*}-\hat{2}, \mathrm{~b}}}^{\text {new }}$ at the step of contraction described in Eq. ([2.12).

We saw a half coarse-graining step of the Grassmann part. Similarly, one can take the coarsegraining along the $\hat{2}$-direction. Then the scales of the $\hat{1}$-direction and the $\hat{2}$-direction become equal and one can reach large space-time volume by iterating the same steps.

\section{Numerical results}

\subsection{Two dimensional GHOTRG}

Figure $\square$ shows the fermion number density

$$
n=\frac{1}{V} \frac{\partial \ln Z}{\partial \mu}
$$

of the free fermion system and the Thirring model whose tensor network representation is derived in Ref. [可]. In the large $\mu$ region, the fermion number density reaches the saturation density. This is very obvious result. When the coupling constant $g$ is finite, the Silver Blaze like phenomenon occurs at smaller $\mu$, and the fermion number density does not change. This result is consistent with the previous one using the GTRG in Ref. [1]]. If $g=0$, one can calculate exactly the determinant of the Wilson-Dirac operator and the number density of the the free fermion system. Comparing it with the GHOTRG result, we can see the error of GHOTRG is less than 5\% though the definition of the number density includes the first derivative of the free energy. 
Figure [3] shows the eigenvalues of $M^{ \pm}$which are made from coarse-grained tensors. The hierarchy of eigenvalues at $\mu=1.0$ is clearly gentler than that at $\mu=2.0$, and it gets worse as the number of iteration increases. This behavior is due to the fact that the accuracy of (HO)TRG becomes worse near the critical point or the cross-over point.

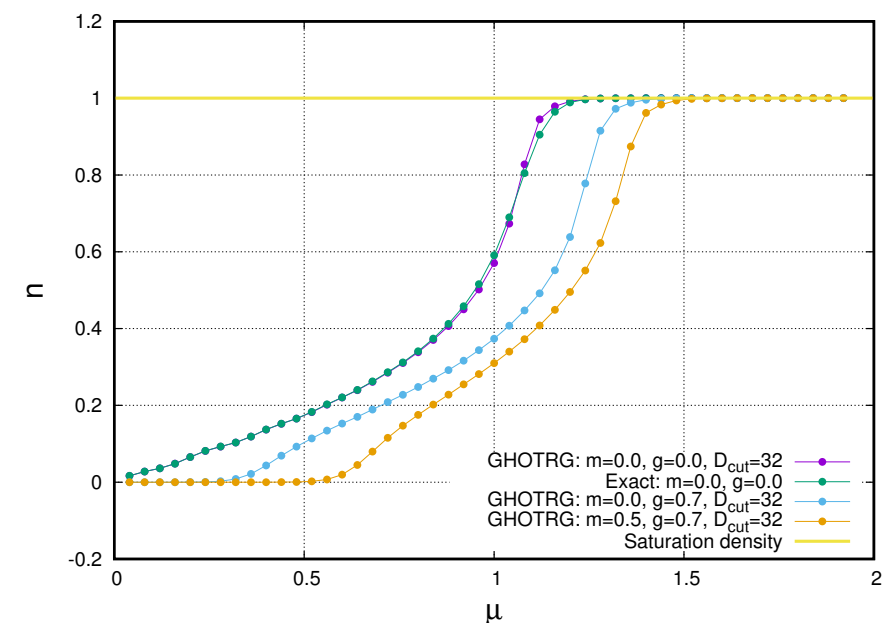

Figure 2: The fermion number density. For massless free case, the exact value is also shown. $V=32 \times 32$.

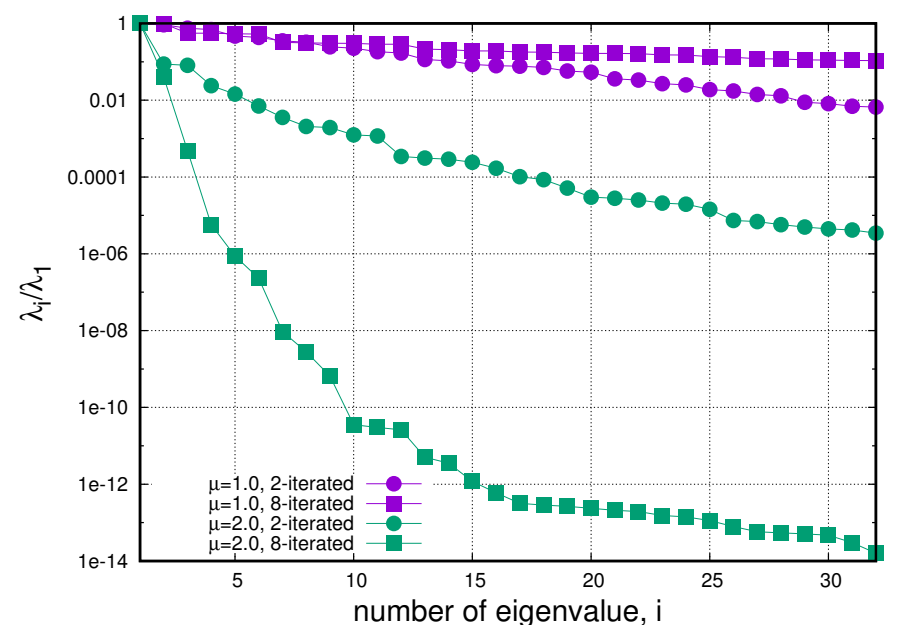

Figure 3: The eigenvalues of $M^{ \pm}$for massless free fermion. $\mu=1.0,2.0, D_{\text {cut }}=32$.

\subsection{Three dimensional GHOTRG}

We apply GHOTRG to the three dimensional free fermion system to calculate the free energy. On account of computational limitations, we carried out the calculation up to $D_{\text {cut }}=6$. Figure 4 shows the relative error of the free energy defined by

$$
\delta=\frac{\ln Z_{\text {Exact }}-\operatorname{Re} \ln Z\left(D_{\text {cut }}\right)}{\left|\ln Z_{\text {Exact }}\right|} .
$$


When we set $D_{\text {cut }}=6$, the percent of accuracy is around $4 \%$ for $8 \times 8 \times 8$ lattice. The reader may feel that $D_{\text {cut }}=6$ is very rough approximation. Actually we observe that when $D_{\text {cut }}$ is small, say 2, 4 and 6, the accuracy of the calculation is not monotonically improved for increasing $D_{\text {cut }}$.

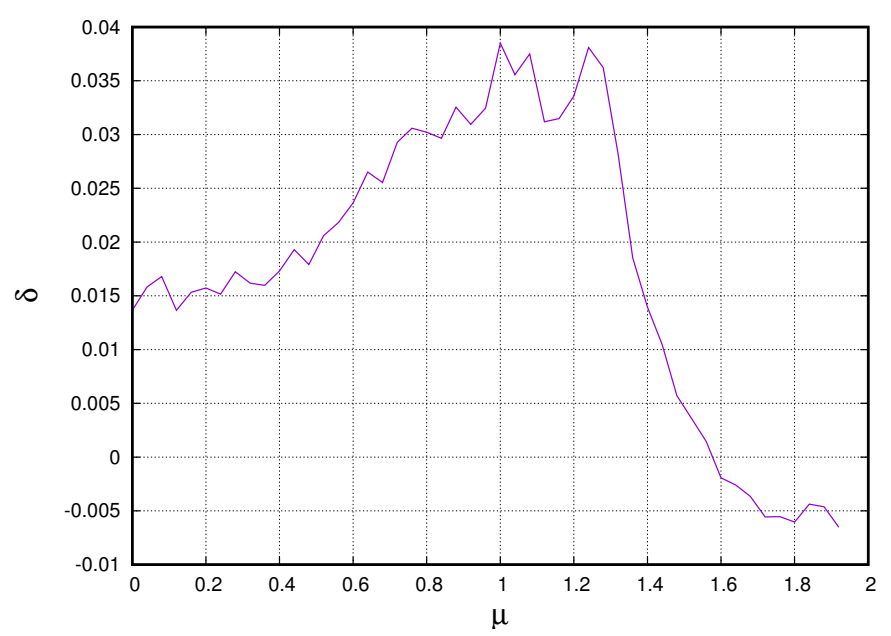

Figure 4: The relative error of the free energy. $V=8 \times 8 \times 8, m=0.0, g=0.0, D_{\text {cut }}=6$.

\section{Summary and outlook}

We formulated Grassmann higher order tensor renormalization group and applied it to the free fermion system and the Thirring model. The results are consistent with analytical or previous ones thus we conclude that GHOTRG is a correct algorithm.

Observing the hierarchy of eigenvalues, the accuracy of GHOTRG seems to become worse around the transition point $(\mu \approx 1)$. This shows that the situation of accuracy for GHOTRG is similar to that of the GTRG.

In principle, now one can deal with relativistic fermion systems in any dimension by using GHOTRG. It is, however, not immediately possible to start four dimensional QCD with finite fermion density or $\theta$ term owing to a huge computational cost. Therefore first it is legitimate to study lower dimensional systems suffering from the sign problem, such as lattice chiral gauge theories, lattice SUSY and so on. We hope that in future our formulation will be used in such studies.

\section{References}

[1] M. Levin and C. P. Nave, Phys. Rev. Lett. 99, 120601 (2007), [arXiv:cond-mat/0611687].

[2] Z. Y. Xie et al., Phys. Rev. B 86, 045139 (2012), [arXiv:1201.1144 [cond-mat.stat-mech]].

[3] Y. Liu et al., Phys. Rev. D88, 056005 (2013), [arXiv:1307.6543 [hep-lat]].

[4] Y. Shimizu and Y. Kuramashi, Phys. Rev. D 90, 014508 (2014), [arXiv:1403.0642 [hep-lat]].

[5] S. Takeda and Y. Yoshimura, PTEP 2015, 043B01 (2015), [arXiv:1412.7855 [hep-lat]]. 